\title{
Animal models of Alzheimer's disease embrace diversity
}

\author{
Alzheimer's disease remains untreatable, despite decades of preclinical research to understand it better and find \\ therapeutic targets. New ways of thinking about a variety animal models are rising to the challenge.
}

\author{
Ellen P. Neff
}

A lzheimer's disease $(\mathrm{AD})$ has long been thought of as somewhat of a monolithic affliction, "this one umbrella that's mainly characterized by amyloid plaques and neurofibrillary tangles of tau," says Kristen Onos, a research scientist at The Jackson Laboratory. "That's what Alzheimer's disease is: end of story."

That story, however, is changing; as human datasets grow, patients are identified earlier in the course of their disease, and computational biology starts to tackle all that human data, it's becoming apparent that there is not necessarily one definition of AD. Amyloid beta plaques and tau tangles might only be partially responsible for the disease, while vascular and immune system contributions might be much more important than previously recognized. "We're seeing a lot of mixed pathologies," says Onos.

With the burden of the disease only expected to grow in coming years and little but clinical failures to report so far in terms of treating it, the preclinical field is starting to look in new directions. Many of the current mouse models, for example, were made to model mutations in genes associated with early onset, familial forms of $\mathrm{AD}$, which afflicts people before the age of 65 but represents only about $5 \%$ of the total Alzheimer's patient population. To develop models for the other $95 \%$, the National Institutes of Health National Institute of Aging (NIH NIA) launched the MODEL-AD program, a consortium of researchers from Indiana University, The Jackson Laboratory, Sage Bionetworks, and the University of California Irvine, in 2016 to focus on lateonset Alzheimer's research and encourage the development of more translationally relevant animal models. "Animal models of $\mathrm{AD}$ are very high on our list of priorities, so there will be more initiatives coming," says Lorenzo Refolo, the director for AD drug discovery and development at NIA.

Mice will likely not stand alone. "There's no perfect model for any disease; the only perfect model would be another human being, but of course that wouldn't work," says NIA's Manuel Moro. "Each model has

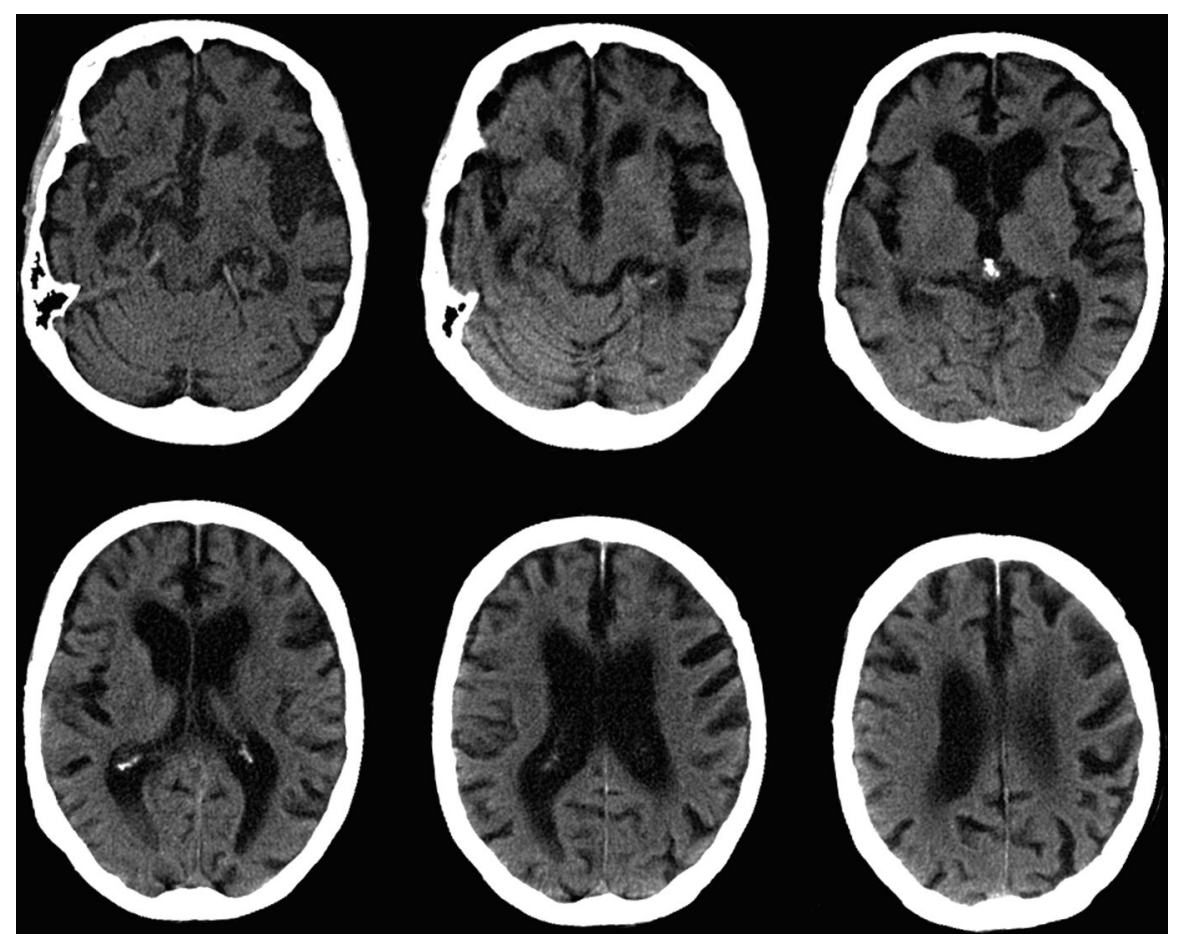

Alzheimer's on the brain. A CT-scan of an Alzheimer's patient. Researchers are looking for new and better ways to model the disease in animals. Credit: Science Photo Library / Alamy Stock Photo

advantages or disadvantages depending on what you're evaluating, depending what you're studying, depending what you're testing," he continues. "In fact, you may have to use a combination of models to study a particular area of Alzheimer's disease."

In the murine world, some researchers are introducing more genetic diversity into mouse experiments to better reflect the heterogeneity found in people. Years of following man's closest relatives are yielding insight into the natural progression of neurodegeneration. Others still have been taking discovery outside the realm of mammals entirely. "When you consider what our population, what our society is facing, as we are all getting older and living longer," says Guy Caldwell, a Caenorhabditis elegans researcher at The University of Alabama, "we need an all-hands-on-deck approach."
Diversifying from 'mouse' to 'mice' When it comes to $\mathrm{AD}$, some might say that mice are furry little failures, with decades of research passing through the laboratory mouse without translational success in the clinic. Rodents do not naturally develop the defining neuropathologies associated with $\mathrm{AD}$, though they can be engineered to express the offending proteins such as amyloid beta, a contributor to plaques, or tau, a protein that causes neurofibrillary tangles in the human brain.

"I wouldn't characterize the models that we have as failures," says Brian Kraemer, an AD scientist at the University of Washington. Rather, he argues that they may have just been mis-used. "They have plaques but no tangles, or they have tangles but no plaques, and most of the models have very little neurodegeneration," he says. 


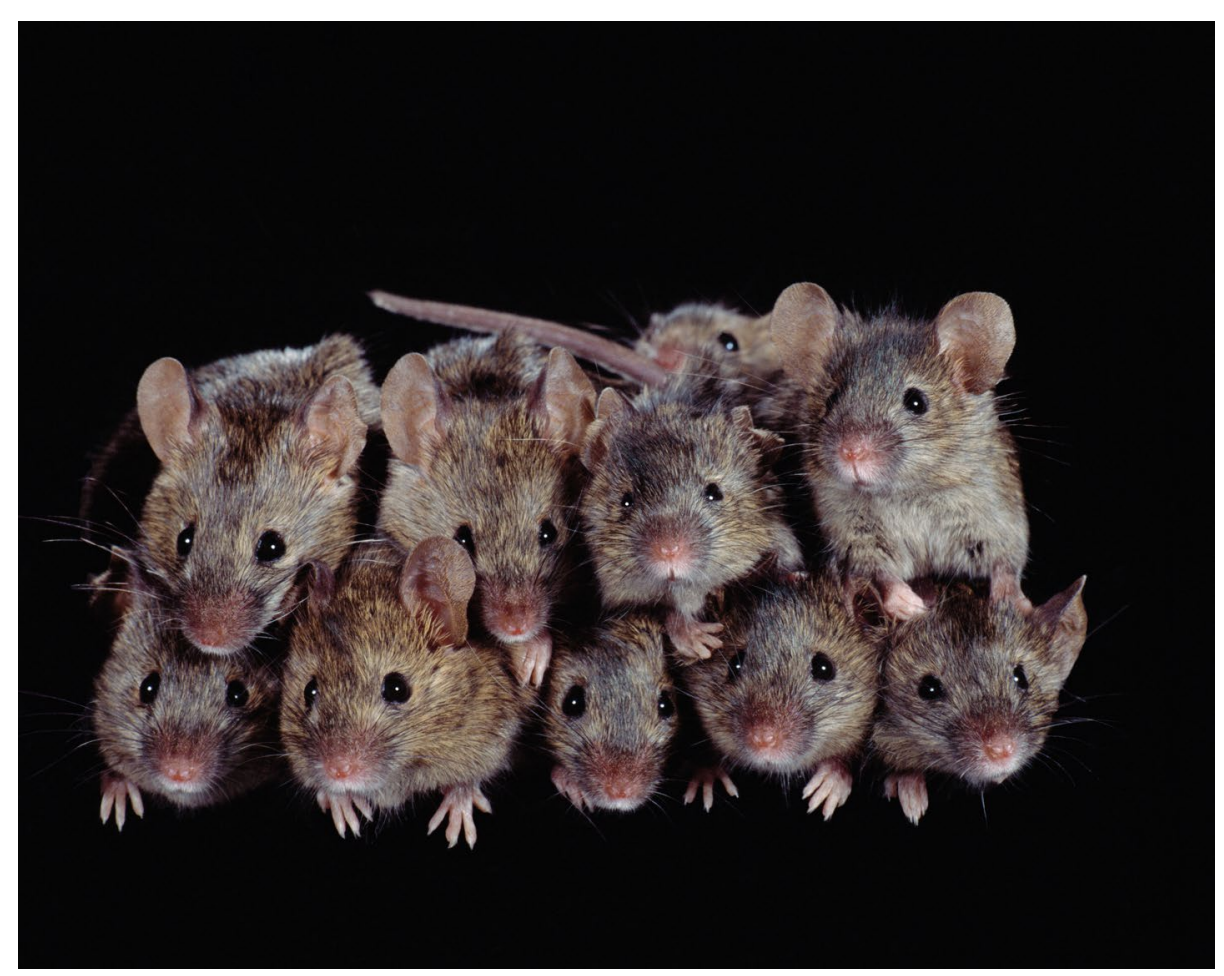

More mice! Laboratory mouse strains are all the same species, Mus musculus. Adding more genetic diversity into house mouse models might be capture the diversity inherent in human patients. Credit: Renaud Visage / Photographer's Choice RF / Getty

"Predicating a clinical trial on the basis of an incomplete model of the disease is problematic. So that's not a failing of the model, per se-it's an over-interpretation of the model." The complete cascade-plaques driving tangles driving neuron loss-still needs to be captured, he says. "I think the field is very much still working towards that."

Mouse work continues on, with some groups considering the most basic of details: what mouse are you building your model with? "We don't think it has to do with the species," says The Jackson Laboratory's Catherine Kaczorowski. "We think has to do with using only one background strain." The background strain of many a mouse model of $\mathrm{AD}$ is the $\mathrm{C} 57 \mathrm{BL} / 6 \mathrm{~J}$ mouse. This strain, however, appears to be particularly resistant to $\mathrm{AD}$-resembling neuropathologies. Drawing conclusions from a just single strain can thus be fraught, so researchers such as Kaczorowski and Onos, motivated by the increasing diversity observed in human disease, are introducing more diversity into their mouse work.

"The idea of using different mouse strains is not a new one to the field of genetics and certainly not in the field of neuroscience, but it hasn't been really widely adopted until just recently," says Onos. Her approach to the 'Black 6' problem has been to go wild. Conventional lab mice strains are all the same species: Mus musculus. However, there are actually three distinct subspecies of the house mouse, which researchers from The Jackson Laboratory sampled and established as inbred strains in the 1980s: CAST mice originally came from Thailand; PWK hail from the Czech Republic; and those known as WSB were found on the eastern shore of Maryland. Compared to the C57BL/6J, the genomes of these mice carry a number of different single nucleotide polymorphisms, similar to those present in human populations, says Onos.

As part of Gareth Howell's lab and the MODEL-AD consortium, she's been placing genes carrying mutations that cause a rare form of familial AD_known as APP and PS1-into these wild-derived mice and characterizing the resulting variation as the animals naturally age. Recently published work ${ }^{1}$ revealed a number of differences between the strains, including in risk factors such as activity, circadian rhythms, and even neuroinflammatory responses, increasingly recognized as a critical component of $\mathrm{AD}$ in people, Onos says. "We were not expecting to find such striking differences between the wild-derived strains."

Working with 'wild' mice meant starting a bit from scratch, as many assays have been validated on young, male Black 6 subjects, Onos says. She and her colleagues had to adapt transgenic techniques and identify appropriate antibodies that would work with the different mice, for example. They also had to figure out how to just keep them in one place in the first place. "The biggest challenge that we found was really the wildness factor," Onos says. Behavioral assays and experimental setups needed to be re-thought and tested with flee-fancy mice in mind. In the end though, she thinks it's been worth the effort. These strains are more human-relevant, she says; from here, they want to assess the role of both genetic and environmental risk factors. That will include studying the APOE4 gene, the most well-known genetic risk factor at the moment for late onset $\mathrm{AD}$, in these sensitized genetic backgrounds.

Similar themes have emerged from Kaczorowski's lab. They have been breeding the 5XFAD transgenic AD mouse with mice from the BXD panel, a large, fully sequenced and reproducible population of mice originally crossed from the C57BL/6J and the DBA/2J strains that display 5 million segregating genetic polymorphisms. "We are really leveraging the population in order to identify modifiers that promote resilience," she says. Some mice proved susceptible to transgene-produced plaques, while others were less behaviorally and physiologically affected over the course of their lifespans ${ }^{2}$. The team is now working to separate strains via hierarchical clustering into separate groups for further testing. "Some of them might have a really robust neuroinflammatory response; another group might have a really robust neurodegeneration profile; another might have more of a demyelination phenotype," says Kaczorowski. "So the first thing we got to do is figure out these subtypes."

Working with multiple strains means working with a lot of mice. "I think it's very similar to working with any big dataset," Kaczorowski says. They needed to develop appropriate assays and the means to analyze all the resulting data, as well as control for confounding measures of normal agerelated deficits. The sheer number of mice needed might be more than some smaller facilities might be able to afford or manage, Kaczorowski acknowledges, but her lab is biobanking tissues from all those animals for others who might not have the resources to work with such large experimental cohorts directly.

\section{Are the closest brains worth the wait?}

Mice remain invaluable, but their evolutionary distance from man can be a limitation. The structure and neurobiology of their brains differ from that of humans, as can their immune system. Looking beyond mice, some researchers are interested in using 
an animal that might be more translationally relevant to people. "We're all looking for other kinds of models," says Carol Shively at Wake Forest University in North Carolina. "Nonhuman primates seem like a very likely place to look for models simply because their central nervous system is as close to ours as any animal that we can study."

Experimental nonhuman primate models of $\mathrm{AD}$ exist; amyloid beta plaques for example can be induced through inoculations of the protein; transgenic and gene edited animals are also in the works. Marmosets are becoming particularly popular, says Moro. Macaques can be gene edited to an extent, but with the marmoset might be easier to fine tune. "The marmosets are very amenable to those gene editing techniques where you just change a few base pairs in the DNA and then you have a risk variant," says Refolo, "You can recapitulate those late onset risk variants.". The first genetically engineered marmoset models of $\mathrm{AD}$ are expected soon from several research laboratories.

Experimental models-whether artificially induced to display a particular pathology or engineered to express humanrelevant variants- have a molecular advantage. "You can dig down and look at much more fine-tuned mechanisms," says Shively. These can be studied without waiting for the animals, which have considerably longer lifespans than a lab rodent, to grow old. "But, etiology is off the table," she says. How a patient ends up with a disease can however be an important consideration for preventing it in the first place-or at least mitigating its most severe effects. "Once people have Alzheimer's, their brains are so degenerated that coming back from it is unlikely," Shively says, at least with current diagnostic and treatment options. A naturally aged animal, studied over the course its lifespan, can be valuable in that regard.

For a time, $\mathrm{AD}$ was indeed considered a unique disease to humans. "We thought that no nonhuman primates had the neuropathology of Alzheimer's," says Agnès Lacreuse at the University of Massachusetts Amherst. Several species exhibited amyloid beta plaques for example, but not the neurofibrillary tangles made of aggregates of abnormally phosphorylated tau protein, she says. "But in fact, people had not looked in brains that were old enough." While it can certainly take a long time for many nonhumans primates to grow old, evidence of naturally occurring tangles have recently been observed in the brains of very old animals, including chimpanzees ${ }^{3}$ and macaques ${ }^{4}$.

Vervets, an Old World monkey native to Africa, might also be added to the list. Shively currently studies members of a colony of several hundred vervet monkeys that moved to Wake Forest from The University of California Los Angeles in 2008. Family relationships are carefully documented and each animal is genotyped and phenotyped "but beyond that, we try to let them be as much as possible," she says. The vervets, living out their lives with their family groups in enriched enclosures in North Carolina, are part of a longitudinal study aimed at identifying factors early in life that contribute to $\mathrm{AD}$ in order to change the trajectory of disease, rather than attempt to bring patients back from its ravages. The focus, Shively says, is on modifiable risk factors, such as body fat \& cholesterol, hypertension \& heart disease, and depression $\&$ stress. "We can intervene on these things when you're 30 and when you're 40 so that when you're 70 , you aren't diagnosed with $\mathrm{AD}$. So that is the focus of the Alzheimer's Disease Research Center here," she says.

When the animals reach the end of their natural lives - in captivity, their mid-20stheir brains are preserved and placed in a repository. In a recent publication ${ }^{5}$, Shively and her colleagues saw signs of both amyloid plaque \& tau pathology; CSF beta amyloid correlated with both age and amyloid plaque density, suggesting a potential noninvasive biomarker of disease progression. Amyloid and tau burden predicted reduced brain volume and reduced glucose metabolism too. "Those were our initial observations that suggested to us that this might be a really promising early model of early changes associated with AD," says Shively.

Along with that brain data, researchers also have years of animal behavior to compare as well; the team at Wake Forest monitors features such as physical activity and walking speed of the animals (variables known to decline in aging humans) as well as changes in social function (an important consideration for keeping elderly people engaged and active in their communities, Shively says). The brains of the vervets are periodically imaged with MRI by the same techniques used to scan humans, and other means of assessment are in the works. "We're trying to develop cognitive assessments that are not too demanding of the animals," Shively says, acknowledging that stress could very well be risk factor for AD. Traditional assessments require considerable time alone and away from the colony; alternatives in the works would require just 30 testing minutes a day for a few weeks.

A faster aging nonhuman primate option might be found in the marmoset. They are less closely related to humans than other primate species, but they get old in 10 to 12 years rather than 20 or 30 . Marmosets do not show neurofibrillary tangles per se, but they do develop hyperphosphorylated tau that can be considered as a precursor to the tangles, notes Lecreuse. A naturally aging colony is being expanded at the Texas Biomedical Research Center in San Antonio; Lacreuse in Massachusetts has also spent the past few years working with the animals. She initially switched from macaques to the much smaller marmosets in 2011 to study sex differences in cognition as the animals aged but recently received an NIH supplement grant to look at Alzheimer's risk in her aging cohorts. "This is the

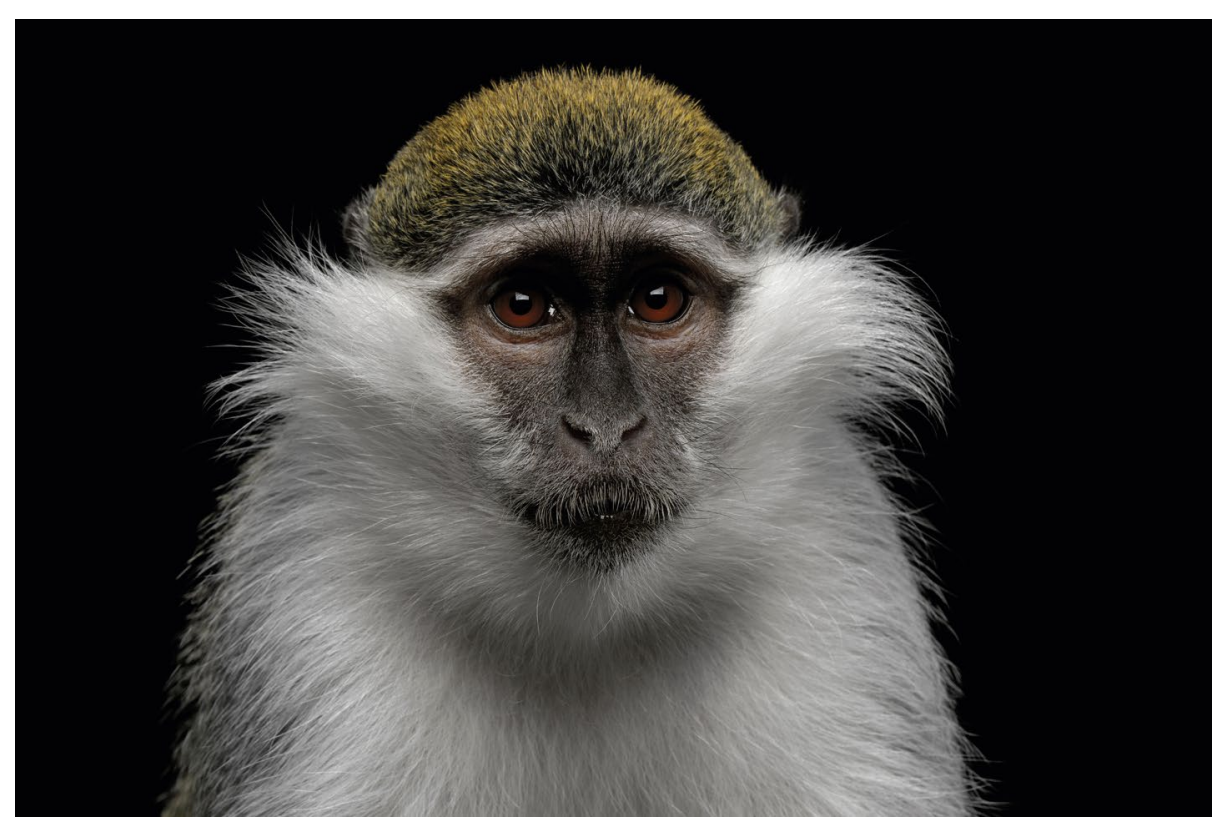

Getting closer. The vervet monkey, Chlorocebus pygerythrus, is one of several nonhuman primate options to study Alzheimer's disease. Credit: Sergey Taran / Alamy Stock Photo 


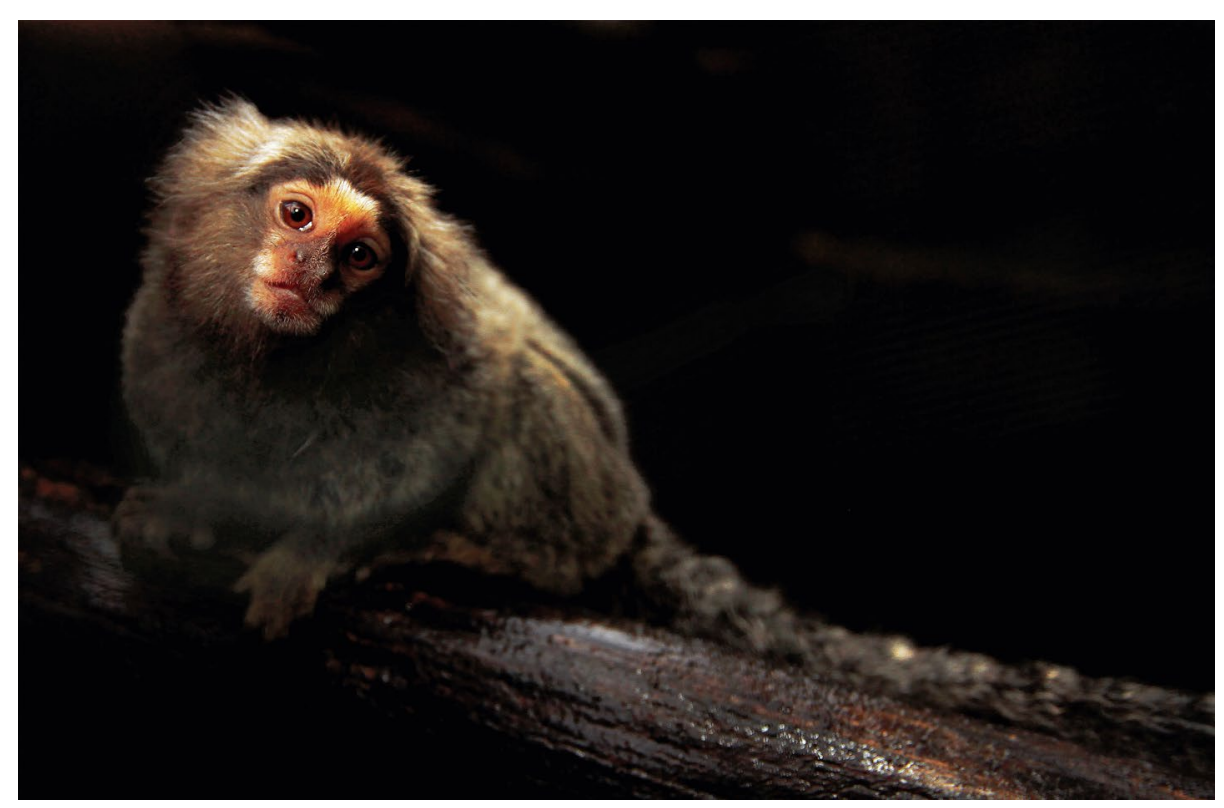

A little bit faster now. The marmoset, Callithrix jacchus, is increasingly popular nonhuman primate for Alzheimer's research. They age more quickly than others. Credit: Arief Prabowo / EyeEm / Getty

beauty of it: the deposition of amyloid and hyperphosphorylated tau is completely natural," says Lacreuse. "I want to look at the progression of the pathology and understand, what are the early symptoms?"

She too is keeping track of behavior, including measures such as memory impairment, sleep changes, and spatial disorientation, to compare with brain tissue after the animals die. A 'fitbit'-like collar to track movement is producing initial data, and she's establishing appropriate equipment and tests to study the marmosets' mental capacities. In the future, she hopes to study sleep disturbance more closely. "Sleep disturbances appear before clinical symptoms; people are thinking that it's actually causal," she says.

As other naturally aged brains are collected, comparisons will be key, says Lacreuse. "If you compare species of primates with different lifespan and different life histories and so on....we can try to understand how it all works." Is it truly a disease of the longest-lived species?

\section{The early worm gets the drug}

For all the genetic predisposition and lifestyle factors that might contribute to an individual's risk of $\mathrm{AD}$, one risk factor indeed looms above them all: age. "The strongest risk factor for essentially all neurodegenerative diseases is age," says Chris Link, a researcher at the University of Colorado Boulder who has long worked outside the mammalian realm. "If you can't replicate age in your model, then you're missing something." A mouse ages faster than a marmoset which ages faster than a macaque, but even the two years it takes for mice to get old can still feel like a long time to some investigators. Invertebrate model systems such as C. elegans worms and Drosophila flies certainly come with caveats, but they might help satisfy a need for speed, especially in terms of identifying potential drugs or therapeutic targets. "This idea that you can only do the discovery aspects of drugs in mammalian systems has cost a lot of time," says Caldwell at Alabama. "And let's face it, when it comes to Alzheimer's, we don't have time."

Though they might be more commonly applied to questions of basic biology, worms aren't necessarily new to the field of disease-specific research: the first $\mathrm{AD}$ model was introduced by Link in $1995^{6}$. His lab developed a transgenic worm that expresses human amyloid beta in the body wall and progressively becomes paralyzed. "The key idea was that if expression of the human peptide gave us phenotypes, then we could do classic genetic screening, looking for suppressor mutations, etc., to identify components of the toxic process," says Link. Other labs have since been introducing different mutations and transgenes to attempt to recapitulate some of the hallmarks of $\mathrm{AD}$.

"It's easy to say we make Alzheimer's worms," says Link, who still works with $C$. elegans models of various neurodegenerative diseases. "But what I try to say more formally is: we make models to test beta amyloid peptide toxicity, because that's really what we're testing." Starting with the right questions is the most important thing, he says. "The worms don't have a brain-it's hard for them to have Alzheimer's." They don't have an adaptive immune system either, nor is it really possible to study physiology in the worm, he says. "It doesn't mean that you can't learn things from your models, but you have to be careful about what you're trying to find out."

What do worms have? Neurons-302, to be exact (in the hermaphrodite; the male worm was recently shown to have 385 neurons). They were the first multicellular animal to be sequenced, their cell lineages and neural connectomes have been mapped, and the animals are self-fertilizing. The worms are also optically transparent-the advent of GFP in the early 2000s meant fluorescent proteins could be expressed and easily observed throughout the animals over the course of their lifetimes. That feature has paired well with improvements in microscopy. "Worms are really good for that, because you have all these fluorescent probes so it's easy to see what's going on at very high, high resolution," says Link. Editing the worm, particularly with the advent of CRISPR, has gotten easier over the years as well. CRISPR has been an accelerant in mouse research, says Kraemer, who maintains both worm and mouse models of $\mathrm{AD}$ with an interest in tau in his lab, but it's been more catalytic for working with worms. "You can now do a whole bunch of things you could only really dream about previously," he says.

Notably for diseases of age, worms get old fast. They age in a matter of days, rather than months or years, thus lending themselves to highly controlled, high throughput and fast studies-all at lower cost than other animals. "Some people need an entire grant just to fund their mouse colony," says Caldwell. "I believe the model systems can really help us in terms of narrowing down what genes, what drugs, what targets might be worth taking forward, instead of using the mammalian system as a screen, or as an original test."

Success in the worm won't necessarily, or directly, mean success in people. They are bit of a blank slate says Caldwell, which can be both good and bad. "Worms don't have amyloid beta peptide, and they don't have APOE, for example," he says. Introducing human genes means they can be studied without interference from endogenous proteins, but, given that they are absent they might not function the same way in the animal in the first place. "It's all about discovery, but you still have to validate," says Caldwell. "You do have to demonstrate that... the readout that you're looking at-be it paralysis, be it degeneration-that you're finding things that are also found in the mammalian systems." 


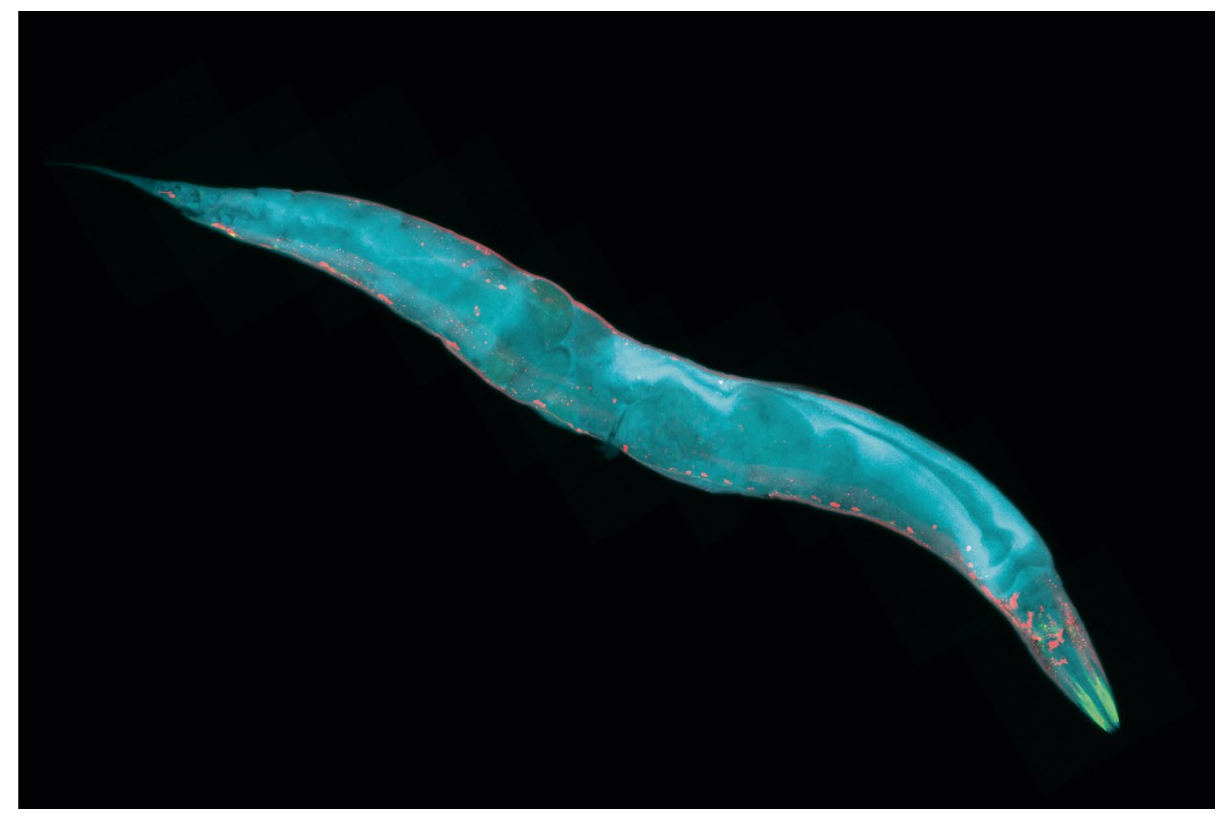

Moving beyond mammals. C. elegans might not have a brain but it ages quickly and is amenable to genetic manipulation and high-throughput experimental approaches. Credit: HeitiPaves / iStock

Worms are far removed evolutionarily from humans, but, notes Kraemer "the evolutionary distance between a worm and a mouse is enormous compared to the evolutionary distance between a mouse and a human...if it's true in a worm and it's true in a mouse, I think it's very likely to be true in you and I as well."

Indeed, "humanized" worm models where amyloid beta, tau, or APOE genes ${ }^{7,8}$ have been introduced do recapitulate some of the age-associated neurodegeneration of
$\mathrm{AD}$, notes Caldwell; some genetic modifiers identified in worm have translated to mammalian models, and are even reflected in human patient genomes.

\section{Variety is the spice...}

As they work with familiar, and less familiar, animal models, many researchers are indeed looking at a wider array of variables beyond those that have long been the focus and defining characteristics of AD. "People are starting to move away from amyloid

\section{Box 1 | To be, or not to be...biased}

When working with animal models, is better to look from man to animal, or from animal to man?

"We definitely pay attention to the human disease literature, a variety of different clinical collaborators to keep us on the translational straight and narrow," says Kraemer. "Being a molecular person you can get kind of distracted from the real disease sometimes." That said, he prefers approaching the worms in an unbiased fashion. "We do a lot of discovery work in the C. elegans and the things that seem the most promising we try and translate into mouse models or study in human tissue."

As human data grows, more targeted approaches to model particular risk or resilience genes could be of value too. "How many lists of genes can human immunization-that's going to be huge," says Kaczorowski. Instead of focusing on one just one element of the disease, groups are looking to different targets to try and accelerate the pace of research and its translation into the clinic. "I think the openness to look beyond the classic pathologies is awesome," she says.

There are more than just mental impairments to consider too. Kaczorowski's collaborator Kristen O’Connell, for example, is interested in the non-cognitive changes that come with $\mathrm{AD}$ that influence quality of life, such as changes in sleep and circadian rhythm, fragility, and motor function that may or may not be impaired (or improved) by the same pathways involved in cognition and memory. "You might end up with a patient who, maybe their memory is improved and so those aspects get better, but they still have a hard time living on their own," O'Connell says. "Then it's important to understand the difference from normal aging, because all these things happen as we age, too...Is it representing an accelerated aging process, or is it really a unique process to Alzheimer's disease-related dementia?"

In the end, with a disease as complex Alzheimer's there are many complex factors to consider in many different ways (Box 1). "Everybody brings a unique perspective and a unique approach," says Shively, noting the increase in nonhuman primate papers in the past decade as well as attention paid to novel strategies by organizations such as the NIA. "I think that the scientific community is on board for new ways to address this problem."

Just as there may not be one single Alzheimer's disease, one animal alone might not lead to its cure. "Having a variety is important, because you don't know which model is going to give you the breakthrough," says Caldwell. "You can guess and obviously say, this is closer to a human, but how many times have mice not reproduced in human?" But, a word of caution as models of all species proliferate. "You still have to really find which ones are telling you something translationally," says Caldwell. "Anyone can create a modeldoes it reflect what's happening?"

\section{Ellen P. Neff \\ Lab Animal. \\ e-mail:ellen.neff@us.nature.com}

Published online: 19 August 2019 https://doi.org/10.1038/s41684-019-0377-8

\section{References}

1. Onos, K. D. et al. PloS Genet 15, e1008155 (2019).

2. Neuner, S. M., Heuer, S. E., Huentelman, M. J., O'Connell, K. M. S. \& Kaczorowski, C. C. Neuron 101, 399-411 (2018).

3. Edler, M. K. et al. Neurobiol Aging 59, 107-120 (2017).

4. Paspalas, C. D. et al. Alzheimers Dement 14, 680-691 (2017)

5. Latimer, C. S. et al. Alzheimers Dement 15, 93-105 (2019).

6. Link, C. D. Proc Natl Acad Sci USA 92, 9368-9732 (1995)

7. Fang, E.F. et al. Nat Neurosci 22, 401-412 (2019).

8. Griffin, E.F. et al. Dis Models Mech 12, dmm037218 (2019). 